\title{
LMI based Stability Analysis and Controller Design for a Class of 2D Continuous-Discrete Linear Systems
}

\author{
K. Galkowski ${ }^{1}$, W. Paszke ${ }^{1}$ B. Sulikowski ${ }^{1}$, E. Rogers ${ }^{2}$, D. H. Owens ${ }^{3}$ \\ 1 Institute of Control and Computation Engineering, University of Zielona \\ Gora, Zielona Gora, Poland. \\ ${ }^{2}$ Dept. Electronics and Computer Science, University of Southampton, \\ Southampton SO17 1BJ, U.K. \{etar\}oecs.soton.ac.uk \\ ${ }^{3}$ Dept Automatic Control and Systems Engineering, University of Sheffield, \\ Sheffield S1 3JD, U.K.
}

\begin{abstract}
Differential linear repetitive processes are a distinct class of $2 \mathrm{D}$ continuous-discrete linear systems of both applications and systems theoretic interest. In the latter area, they arise, for example, in the analysis of both iterative learning control schemes and iterative algorithms for computing the solutions of nonlinear dynamic optimal control algorithms based on the maximum principle. Repetitive processes cannot be analysed/controlled by direct application of existing systems theory and to date there are few results on the specification and design of control schemes for them. This paper uses an LMI setting to develop the first really significant results in this problem domain.
\end{abstract}

\section{ACC02-IEEE1301}

\section{Introduction}

The essential unique characteristic of a repetitive, also termed multipass in the early literature, process can be illustrated by considering machining operations where the material or workpiece involved is processed by a sequence of passes of the processing tool. Assuming that the pass length $\alpha$ (i.e. the duration of a pass of the processing tool) is finite and constant, the output vector, or pass profile, $y_{k}(t), 0 \leq t \leq \alpha$ ( $t$ being the independent spatial or temporal variable) produced on the $k$ th pass acts as a forcing function on the next pass and hence contributes to the dynamics of the new pass profile $y_{k+1}(t), 0 \leq t \leq \alpha, k \geq 0$.

Industrial examples of these processes include long-wall coal cutting and metal rolling operations; see $[1,2]$ for a detailed treatment. Also cases exist where adopting a repetitive process setting for analysis has major advantages over alternatives, so-called algorithmic examples. This is especially true for classes of iterative learning control (ILC) schemes [3] and of nonlinear dynamic optimal control problems based on the maximum principle [4]. In the former area, the stability theory for linear repetitive processes proves to be a natural setting for the analysis of a powerful class of control laws in a subject area which has seen many successful practical implementations.

The basic unique control problem for repetitive processes is that the output sequence of pass profiles can contain oscillations that increase in amplitude in the pass-to-pass direction (i.e. in the $k$ direction in the notation for variables used here). Such behavior is easily generated in simulation studies and on scaled models of industrial examples such as long-wall coal cutting (see [1] for a detailed treatment). Also these processes cannot be stabilized/controlled, in all but a few very restrictive special cases, by application of standard (or 1D) control systems techniques (such an approach would essentially ignore their key defining feature and the effects of resetting the initial (or boundary) conditions before the start of each new pass). (Elements of 1D linear systems theory, such as stability tests, can, of course, be employed where appropriate/relevant.)

To remove these deficiencies and provide the essential starting point for a comprehensive control/systems theory for these processes, a rigorous stability theory has been developed $[5,6]$. This theory is based on an abstract model of the dynamics in a Banach space setting which includes all processes with linear dynamics and a constant pass length as special cases. Also the results of applying this theory to a range of sub-classes have been reported - including the differential linear repetitive processes considered here where the resulting conditions can be tested by direct application of $1 \mathrm{D}$ linear systems stability tests, e.g. in 
the frequency domain.

Unlike the 1D linear systems case, however, the 'Nyquist-like' stability tests which can be used in this case do not provide any measures of relative stability or form the basis for systematic control systems design. In actual fact, little is yet available in this general area. This paper shows that using an LMI setting enables very significant progress to be achieved in terms of the specification and computation of stability margins and systematic algorithms for the design of a (potentially) very powerful class of control laws. We begin in the next section by giving the required background definitions and results.

\section{Background}

The state space model of the differential linear repetitive processes considered here has the following form over $0 \leq t \leq \alpha, k \geq 0$,

$$
\begin{aligned}
& \dot{x}_{k+1}(t)=A x_{k+1}(t)+B u_{k+1}(t)+B_{0} y_{k}(t) \\
& y_{k+1}(t)=C x_{k+1}(t)+D u_{k+1}(t)+D_{0} y_{k}(t)
\end{aligned}
$$

Here on pass $k, x_{k}(t)$ is the $n \times 1$ state vector, $y_{k}(t)$ is the $m \times 1$ pass profile vector, and $u_{k}(t)$ is the $l \times 1$ vector of control inputs.

To complete the process description, it is necessary to specify the 'initial conditions' - termed the boundary conditions here, i.e. the state initial vector on each pass and the initial pass profile. Here no loss of generality arises from assuming $x_{k+1}(0)=0, k \geq 0$, and $y_{0}(t)=f(t)$, where $f(t)$ is an $m \times 1$ vector whose entries are known functions of $t$.

The stability theory $[5,6]$ for linear repetitive processes is based on an abstract model of the underlying dynamics in a Banach space setting which includes all such processes as special cases. In effect, this consists of two distinct concepts termed asymptotic stability and stability along the pass respectively where the former is a necessary condition for the latter. Noting again the unique control problem for these processes, asymptotic stability demands that bounded input sequences (pass initial conditions, disturbances and control inputs) produce bounded sequences of pass profiles (in a well defined sense) over the finite pass length and stability along the pass demands that this property holds independent of this parameter. Hence, in general, it will be the stronger property of stability along the pass which will be required.

Several equivalent sets of necessary and sufficient conditions for stability along the pass of processes of the form defined by (1) have been reported $[5,6]$ but here it is the following set which will be required.

Theorem 1 Suppose that the pair $\left\{A, B_{0}\right\}$ is controllable and the pair $\{C, A\}$ is observable. Then a differential linear repetitive process of the form (1) (with the assumed boundary conditions) is stable along the pass if, and only if, $r\left(D_{0}\right)<1$, all eigenvalues of $A$ have strictly negative real parts, and all eigenvalues of the transfer function matrix

$$
G(s)=C\left(s I_{n}-A\right)^{-1} B_{0}+D_{0}
$$

have modulus strictly less than unity $\forall s=\imath \omega, \omega \geq$ 0 .

\section{LMI based Stability Analysis}

The starting point here is the third condition of Theorem 1. In particular, if the other conditions of this result hold then the example under consideration is stable along the pass if, and only if,

$$
\operatorname{det}\left(z I_{m}-\left(C\left(s I_{n}-A\right)^{-1} B_{0}+D_{0}\right)\right) \neq 0 \text {, }
$$

$|z| \geq 1, s=3 \omega$. Also we will require the following well known definition and result.

Definition 1 Let $S(\gamma)$ be a square matrix of real rational functions in the complex variable $\gamma$. Then this matrix is said to be strictly continuous bounded real if, and only if,

(a) all poles of $S(\gamma)$ have strictly negative real parts; and

(b) $I-S^{T}(-z \omega) S(w \omega)>0, \forall \omega$.

Note. In this paper the symbols $>, \geq$, and $<$ will be used to denote positive definite, positive semi definite and negative definite properties respectively of matrices.

The conditions under (a) and (b) in this last definition can be reduced to conditions on the matrices of a minimal state space realization of $S(\gamma)$. In particular, suppose that $S(\gamma)$ has a minimal state space realization given by the quadruple $\{F, G, H, J\}$ such that

$$
S(\gamma)=H^{T}(\gamma I-F)^{-1} G+J
$$

Then the following result is the well known so-called strictly continuous bounded real lemma(SCBR).

Lemma 1 Suppose that $S(\gamma)$ is a square matrix of real rational functions in $\gamma$ with minimal state space realization defined by $\{F, G, H, J\}$. then this 
matrix is $S C B R$ if, and only if, $\exists$ a positive definite symmetric matrix $P$ such that

$$
Q:=\left[\begin{array}{cc}
F^{T} P+P F+H^{T} H & P G+H J \\
G^{T} P+J^{T} H^{T} & J^{T} J-I
\end{array}\right]
$$

In what follows, we use the SCBR property to show that a solution of the following 2D Lyapunov equation is a sufficient condition for stability along the pass of processes described by (1).

$$
\widetilde{A}^{T} W^{1,0}+W^{1,0} \tilde{A}+\widetilde{A}^{T} W^{0,1} \tilde{A}-W^{0,1}=-Q
$$

where $W^{1,0}=\operatorname{diag}\left\{W_{1}, 0_{m \times m}\right\}$, with $W_{1}=$ $W_{1}^{T}$ an $n \times n$ positive definite matrix, $W^{0,1}=$ $\operatorname{diag}\left\{0_{n \times n} W_{2}\right\}$, with $W_{2}=W_{2}^{T}$ an $m \times m$ positive definite matrix, $Q=Q^{T}$ an $(n+m) \times(n+m)$ positive definite matrix, and

$$
\tilde{A}=\left[\begin{array}{ll}
A & B_{0} \\
C & D_{0}
\end{array}\right]
$$

Now we have the following theorem and corollary from $[5,6]$.

Theorem 2 Consider a differential linear repetitive process of the form (1). Then $\exists$ symmetric positive definite matrices $W_{1}, W_{2}$, and $Q$ such that $W=\operatorname{diag}\left\{W_{1}, W_{2}\right\}$ and $Q$ satisfy the $2 D$ Lyapunov equation (6) if, and only if, $\exists$ an $m \times m$ nonsingular matrix $T$ such that $\tilde{G}(s)$ defined by

$$
\bar{G}(s)=T G(s) T^{-1}
$$

is $S C B R$.

Corollary 1 Consider a differential linear repetitive process of the form (1). Then this process is stable along the pass if $r\left(D_{0}\right)<1$, all eigenvalues of the matrix $A$ have strictly negative real parts and $\exists$ positive definite symmetric matrices $W_{1}$, $W_{2}$ and $Q$ which solve the $2 D$ Lyapunov equation (6).

In general, the reverse conclusion to this last corollary is not true - a counter-example can be found in [6]. If, however, the example under consideration is single-input single-output then the result of this corollary is necessary and sufficient.

To develop an LMI solution of the 2D Lyapunov equation here, first note that (6) can be rewritten in the form

$$
\widetilde{A}_{2}^{T} \widetilde{W}_{2} \widetilde{A}_{2}^{T}-W^{0,1}+\widetilde{A}_{1}^{T} W^{1,0}+W^{1,0} \widetilde{A}_{1}<0
$$

where $\widetilde{W}_{2}=\operatorname{diag}\left\{W_{3}, W_{2}\right\}, W_{3}$ is an arbitrary symmetric positive definite $n \times n$ matrix and

$$
\tilde{A}_{1}=\left[\begin{array}{cc}
A & B_{0} \\
0 & 0
\end{array}\right], \tilde{A}_{2}=\left[\begin{array}{cc}
0 & 0 \\
C & D_{0}
\end{array}\right]
$$

Now apply the Schur complement and then pre and post-multiply (9) by the matrix $\operatorname{diag}\left\{I_{n}, \vec{W}_{2}\right\}$ to yield the equivalent condition

$$
\left[\begin{array}{cc}
-W^{0,1}+\tilde{A}_{1}^{T} W^{1,0}+W^{1,0} \tilde{A}_{1} & \widetilde{A}_{2}^{T} \widetilde{W}_{2} \\
\widetilde{W}_{2} \tilde{A}_{2} & -\widetilde{W}_{2}
\end{array}\right]<0
$$

which is clearly in the LMI form and we have the following result.

Theorem 3 A differential linear repetitive process of the form (1) is stable along the pass if the LMI of (11) is feasible.

\section{Stability Margins}

First note that in the $1 \mathrm{D}$ differential linear systems case the Lyapunov equation for stability of the system $\dot{x}(t)=A x(t)+B u(t)$ can be written in LMI form as $\exists P>0$, : $A P+P A^{T}<0$. Also the stability margin in this case can be defined as the maximum positive real number $\sigma$ such that $(\sigma I+A)$ has all its eigenvalues in the open lefthalf of the complex plane. A lower bound for $\sigma$ in this case can be obtained from the constrained optimization problem:

Maximize $\sigma$ subject to $P>0, \sigma>0$ and the LMI

$$
A P+P A^{T}+2 \sigma P<0
$$

In the discrete 1D linear systems case, the Lyapunov equation for the system $x(i+1)=A x(i)+$ $B u(i)$ can be written in LMI form as $\exists P>0$, : $A^{T} P A-P<0$. Also the stability margin in this case can be defined as the maximum positive real number $\sigma$ such that $C(z):=\operatorname{det}[I-z A] \neq 0$ in $U_{\sigma}^{1}=\{z:|z| \leq 1+\sigma\}$. To formulate this as an LMI problem, first note that this last condition is equivalent to finding the maximum value of $\sigma \in \mathbb{R}: \bar{C}(z):=\operatorname{det}[I-(1+\sigma) z A] \neq 0$ in $U_{1}=\{z:|z| \leq 1\}$. Routine analysis then leads to the following LMI formulation of this problem: Maximize $\sigma \in \mathbb{R}$ subject to $P=P^{T}>0$ and the LMI

$$
\left[\begin{array}{cc}
-P & (1+\sigma) A^{T} P \\
(1+\sigma) P A & -P
\end{array}\right]<0
$$

In the case of the differential linear repetitive processes, the generalized stability margin is defined as follows, where the fact that (14) below with $\sigma_{n}=0$ is equivalent to the conditions of Theorem 1 has been established in [6].

Definition 2 The generalized stability margin $\sigma_{\eta}$ for a differential linear repetitive process of the form (1) is defined as follows where $0 \leq \eta \leq 1$

$$
C(s, z) \neq 0 \text { in } \widehat{U}_{s, z}^{2}
$$


where $\widehat{U}_{s, z}^{2}=\left\{(s, z): \operatorname{Re}(s)>-\sigma_{\eta},|z| \leq 1+\right.$ $\left.(1-\eta) \sigma_{\eta}\right\}$ and

$$
\dot{C}(s, z)=\operatorname{det}\left[s I_{n} \oplus I_{m}-\left[\begin{array}{cc}
A & B_{0} \\
z C & z D_{0}
\end{array}\right]\right]
$$

Note. It is not possible to define a joint $(s, z)$ stability margin due to the fact that the region $\operatorname{Re}(s)>-\sigma_{\eta}$ is not compact.

The following is the first major new result in this paper and gives an LMI solution to the computation of $\sigma_{\eta}$.

Theorem 4 Lower bounds for $\sigma_{\eta}$ can be obtained as the solutions of the constrained optimization problem:

Given $0 \leq \eta \leq 1$, maximize $\sigma_{\eta}$ subject to $W_{1}>$ $0, W_{2}>0, W_{3}>0, \sigma_{\eta}>0$ and the $L M I$

$$
\begin{gathered}
{\left[\begin{array}{cc}
H & H_{1} \\
H_{1}^{T} & -\widetilde{W}_{2}
\end{array}\right]<0} \\
H=2 \eta \sigma_{\eta} W^{1,0}-W^{0,1}+\widetilde{A}_{1}^{T} W^{1,0}+W^{1,0} \tilde{A}_{1} \\
H_{1}=\left[1+(1-\eta) \sigma_{\eta}\right] \widetilde{A}_{2}^{T} \widetilde{W}_{2}
\end{gathered}
$$

Proof. First note again that (11) is a combination of the Lyapunov equations for differential and discrete 1D linear systems. The result now follows immediately on applying the LMI formulations of the stability margins for differential and discrete linear systems summarized above as (12) and (13) respectively to (11).

One advantage of this latter approach is that we can see the 'trade-off' in terms of the value of $\eta$ between the along the pass and pass to pass components of the two stability margins. This is highlighted in the following example.

Example 1 Consider the case when

$$
\begin{aligned}
A & =\left[\begin{array}{rrr}
-0.1831 & 0.0649 & -0.0243 \\
-0.1464 & -0.0648 & -0.2281 \\
0.0536 & 0.0376 & -0.2364
\end{array}\right] \\
B_{0} & =\left[\begin{array}{rrr}
-0.0937 & 0.0916 & 0.0562 \\
-0.2436 & -0.2036 & 0.0543 \\
-0.0580 & -0.2323 & -0.2421
\end{array}\right] \\
C & =\left[\begin{array}{rrr}
-0.2418 & -0.2212 & 0.1088 \\
-0.1550 & -0.0662 & 0.0963 \\
0.0435 & 0.0657 & -0.2080
\end{array}\right] \\
D_{0} & =\left[\begin{array}{rrr}
-0.0228 & -0.1732 & 0.1138 \\
-0.0291 & 0.0878 & -0.0108 \\
-0.0734 & 0.0996 & 0.0274
\end{array}\right]
\end{aligned}
$$

This process is stable along the pass and the following matrices solve the $2 D$ Lyapunov equation expressed in LMI form

$$
\begin{aligned}
& W_{1}=\left[\begin{array}{rrr}
334.6229 & -70.5901 & 55.0677 \\
-70.5901 & 218.9137 & -92.9743 \\
55.0677 & -92.9743 & 367.3537
\end{array}\right] \\
& W_{2}=\left[\begin{array}{rrr}
220.5601 & -22.2878 & 7.4615 \\
-22.2878 & 250.0284 & 19.3149 \\
7.4615 & 19.3149 & 266.0832
\end{array}\right]
\end{aligned}
$$

and $W_{3}=W_{2}$. The following table gives the relevant stability margins

\begin{tabular}{|c|c|c|c|}
\hline$\eta$ & $\sigma_{\eta}$ & $\sigma_{z}$ & $\sigma_{z}$ \\
\hline \hline 0 & 1.7109 & 0 & 1.7109 \\
0.1 & 0.5293 & 0.0529 & 0.4764 \\
0.2 & 0.3188 & 0.0638 & 0.2550 \\
0.3 & 0.2266 & 0.0680 & 0.1586 \\
0.4 & 0.1766 & 0.0706 & 0.1059 \\
0.5 & 0.1438 & 0.0719 & 0.0719 \\
0.6 & 0.1187 & 0.0712 & 0.0475 \\
0.7 & 0.1031 & 0.0722 & 0.0309 \\
0.8 & 0.0935 & 0.0748 & 0.0187 \\
0.9 & 0.0827 & 0.0745 & 0.0083 \\
1.0 & 0.0759 & 0.0759 & 0 \\
\hline
\end{tabular}

Here $\sigma_{s}=\eta \sigma_{\eta}$ is the component of the generalized stability margin referring to the along the pass dynamics and $\sigma_{z}=(1-\eta) \sigma_{\eta}$ that referring to the pass to pass dynamics.

A currently (essentially) open problem is how exactly these stability relate to expected process performance.

\section{LMI based Controller Design}

In terms of the design of control schemes for differential linear repetitive processes, most work to date has been done in the ILC area [3]. Here it has become clear that a very powerful class of control laws results from using feedback action on the current pass augmented by feedforward action from the previous pass. Here we consider a control law of the following form over $0 \leq t \leq \alpha,: k \geq 0$

$u_{k+1}(t)=K_{1} x_{k+1}(t)+K_{2} y_{k}(t):=K\left[\begin{array}{c}x_{k+1}(t) \\ y_{k}(t)\end{array}\right]$

where $K_{1}$ and $K_{2}$ are appropriately dimensioned matrices to be designed. This results in the following condition for closed loop stability along the pass (resulting from interpreting (14) with $\sigma_{n}=0$ closed loop)

$$
C_{c}(s, z) \neq 0,: \forall:(s, z): R e(s)>0,|z| \leq 1
$$


where

$$
C_{c}(s, z):=\operatorname{det}\left[s I_{n} \oplus I_{m}-S\right]
$$

and

$$
S=\left[\begin{array}{ll}
\left(A+B K_{1}\right) & \left(B_{0}+B K_{2}\right) \\
z\left(C+D K_{1}\right) & z\left(D_{0}+D K_{2}\right)
\end{array}\right]
$$

Now introduce the matrices

$$
\widetilde{B}_{1}=\left[\begin{array}{l}
B \\
0
\end{array}\right], \quad \widetilde{B}_{2}=\left[\begin{array}{l}
0 \\
D
\end{array}\right]
$$

Then we have the following which is the second major result in this paper (which results directly from interpreting (11) closed loop).

Theorem 5 Suppose that a differential linear repetitive process of the form described by (1) is subjected to a control law of the form (19). Then the resulting closed loop process is stable along the pass if $\exists$ symmetric matrices $W_{1}>0, W_{2}>0$ and $W_{3}>0$ such that

$$
\begin{gathered}
{\left[\begin{array}{cc}
F_{1} & \left(\widetilde{A}_{2}+\widetilde{B}_{2} K\right)^{T} \widetilde{W}_{2} \\
\widetilde{W}_{2}\left(\widetilde{A}_{2}+\widetilde{B}_{2} K\right) & -\widetilde{W}_{2}
\end{array}\right]<0} \\
F_{1}=-W^{0,1}+\left(\widetilde{A}_{1}+\widetilde{B}_{1} K\right)^{T} W^{1,0} \\
+W^{1,0}\left(\tilde{A}_{1}+\widetilde{B}_{1} K\right)
\end{gathered}
$$

The difficulty with the matrix inequality of Theorem 5 is that it is nonlinear in its parameters. It can, however, be replaced by the following result where each of the inequalities is a strict LMI with a linear constraint which also gives a formula for computing $K$ in (19). This follows by first noting that the above condition can be rewritten (by direct substitution for the relevant sub-matrices) in the following form

$$
\begin{gathered}
{\left[\begin{array}{cccc}
F_{11} & F_{12} & 0 & F_{14} \\
F_{12}^{T} & -W_{2} & 0 & F_{24} \\
0 & 0 & -W_{2} & 0 \\
F_{14}^{T} & F_{24}^{T} & 0 & -W_{2}
\end{array}\right]<0} \\
F_{11}=\left(A+B K_{1}\right)^{T} W_{1}+W_{1}\left(A+B K_{1}\right) \\
F_{12}=W_{1}\left(B_{0}+B K_{2}\right) \\
F_{14}=\left(C+D K_{1}\right)^{T} W_{2} \\
F_{24}=\left(D_{0}+D K_{2}\right)^{T} W_{2}
\end{gathered}
$$

Theorem 6 The condition of Theorem 5 is equivalent to the requirement that $\exists$ symmetric matrices $Y>0, Z>0$, and matrices $N$ and $M$ such that the following LMI holds.

$$
\left[\begin{array}{ccc}
G_{11} & G_{12} & G_{13} \\
G_{12}^{T} & -Z & G_{24} \\
G_{13}^{T} & G_{24}^{T} & -Z
\end{array}\right]<0
$$

$$
\begin{gathered}
G_{11}=Y A^{T}+N^{T} B^{T}+A Y+B N \\
G_{12}=B_{0} Z+B M \\
G_{13}=Y C^{T}+N^{T} D^{T} \\
G_{14}=Z D_{0}^{T}+M^{T} D^{T}
\end{gathered}
$$

Also if (25) holds, stabilizing $K_{1}$ and $K_{2}$ for the control law (19) are given by

$$
K_{1}=N Y^{-1}, K_{2}=M Z^{-1}
$$

Proof: First pre and post-multiply (20) by the matrix $\operatorname{diag}\left(W_{1}^{-1}, W_{2}^{-1}, W_{2}^{-1}, W_{2}^{-1}\right)$ and then note that the third block row and the third block column can be removed from the result without changing the underlying inequality solution. Now introduce the substitutions

$$
Y=W_{1}^{-1}, Z=W_{2}^{-1}
$$

and use (26) to obtain (25).

To illustrate this last result we now give the following example.

Example 2 Consider the model (1) in the case when

$$
\begin{aligned}
& A=\left[\begin{array}{rrr}
0.8 & 0.4 & -0.8 \\
-0.8 & -0.8 & -0.6 \\
0.5 & 0.9 & 0.5
\end{array}\right] \\
& B=\left[\begin{array}{rrr}
-0.4 & 0.6 & 0.1 \\
-0.2 & -0.5 & 0.05 \\
0.2 & -0.2 & -0.2
\end{array}\right] \text {, } \\
& B_{0}=\left[\begin{array}{rrr}
-0.7 & 0.5 & 0.2 \\
-0.2 & -0.8 & 0.3 \\
-0.5 & -0.2 & -0.2
\end{array}\right] \text {, } \\
& C=\left[\begin{array}{rrr}
-0.9 & -0.2 & 0.2 \\
-0.6 & -0.9 & 0.3 \\
0.6 & 0.5 & -0.9
\end{array}\right] \text {, } \\
& D=\left[\begin{array}{rrr}
0.5 & 0.6 & 0.5 \\
0.2 & -0.6 & 0.5 \\
0.4 & 0.2 & -0.25
\end{array}\right] \text {, } \\
& D_{0}=\left[\begin{array}{rrr}
-0.8 & -0.6 & 0.8 \\
-0.8 & 0.8 & -0.5 \\
-0.3 & 0.6 & 0.2
\end{array}\right]
\end{aligned}
$$

This model is both asymptotically unstable (the eigenvalues of $D_{0}$ are $\{-1.0425$,

$0.6213 \pm 0.574 i\}$ ) and unstable along the pass ( $A$ has eigenvalues $\{0.05 \pm 0.8231 i, 0.6\})$. Theorem 6 can be successfully applied here since the LMI of (25) is feasible Also by (26) of Theorem 6 the stabilizing $K_{1}$ and $K_{2}$ for the control law (19) are given by

$$
K_{1}=\left[\begin{array}{rrr}
19.0682 & 3.0264 & 15.0061 \\
-9.9662 & -1.7118 & -7.4517 \\
-6.9497 & -0.2326 & -7.5848
\end{array}\right] \text {, }
$$




$$
K_{2}=\left[\begin{array}{rrr}
-0.0394 & 1.5696 & -0.5988 \\
0.3749 & -0.2424 & -0.7081 \\
1.2621 & -0.4970 & -0.0024
\end{array}\right]
$$

If some prescribed stability margins $\sigma_{1}^{*}, \sigma_{2}^{*}$ are required by the design specification, then Theorem 5 generalizes in a natural manner and gives the following result.

Theorem 7 Suppose that a differential linear repetitive process of the form described by (1) is subjected to a control law of the form (19) and the stability margins $\sigma_{1}^{*}$ and $\sigma_{2}^{*}$ are required. Then the resulting closed loop system is stable along the pass with these stability margins if $\exists$ symmetric matrices $Y>0, Z>0, N$ and $M$ such that the following LMI holds.

$$
\left[\begin{array}{lll}
F_{11} & F_{12} & F_{13} \\
F_{12}^{T} & -Z & F_{23} \\
F_{13}^{T} & F_{23}^{T} & -Z
\end{array}\right]<0
$$

Also stabilizing $K_{1}$ and $K_{2}$ for the control law (19) are again given by (26).

$$
\begin{gathered}
F_{11}=2 \sigma_{1}^{*} Y+Y A^{T}+N^{T} B^{T}+A Y+B N \\
F_{12}=B_{0} Z+B M \\
F_{13}=\left(1+\sigma_{2}^{*}\right)\left(Y C^{T}+N^{T} D^{T}\right) \\
F_{23}=\left(1+\sigma_{2}^{*}\right)\left(Z D_{0}^{T}+M^{T} D^{T}\right)
\end{gathered}
$$

\section{Conclusions}

Differential linear repetitive processes are a distinct class of 2D continuous-discrete linear systems of both applications and systems theoretic interest. In applications, they arise in iterative learning control schemes and in solution algorithms for nonlinear dynamic optimal control algorithms based on the maximum principle. Repetitive processes cannot be analysed/controlled by direct application of existing systems theory and to date there have been few results on the specification and design of control schemes for them. This paper has used an LMI setting to develop the first really significant results in this key area. Starting from an LMI interpretation of a 2D Lyapunov equation based interpretation of stability, a complete design procedure for a class of control laws based on combining feedback control action on the current pass (here state feedback action has been used) combined with feedforward action based on the previous pass profile. (Experience in the iterative learning control application strongly suggests that such control laws are very powerful). The resulting design procedure can be implemented by direct application of standard MATLAB based LMI routines. Also it has been shown that this method leads to easily computed stability margins and extends naturally to the problem of control law design to meet specified stability margins.

\section{References}

[1] Edwards, J. B. 'Stability problems in the control of multipass processes'. Proceedings of The Institution of Electrical Engineers, 121(11):1425-1431, 1974.

[2] Smyth, K. J. 'Computer Aided Analysis for Linear Repetitive Processes', PhD Thesis, University of Strathclyde, Glasgow, UK, 1992.

[3] Owens, D. H., Amann, N., Rogers, E., and French, M. C. 'Analysis of iterative learning control schemes - a 2D systems/repetitive processes approach'. Multidimensional Systems and Signal Processing, 11(1/2), 2000, pp. 125-177.

[4] Roberts, P. D. Numerical investigation of a stability theorem arising from the 2-dimensional analysis of an iterative optimal control algorithm'. Multidimensional Systems and Signal Processing, 11(1/2), 2000, pp 109-124.

[5] Rogers, E. and Owens, D. H. 'Stability Analysis for Linear Repetitive Processes', Springer-Verlag Lecture Notes in Control And Information Sciences Series, Vol 175, Berlin, 1992.

[6] Rogers, E., Galkowski, K. and Owens, D. H. 'Control Systems Theory and Applications for Linear Repetitive Processes', Springer-Verlag Lecture Notes in Control and Information Sciences Series, Berlin, 2002, to appear.

[7] Rogers, E., Lam, J., Galkowski, K., Xu, S., Wood, J. and Owens, D. H. 'LMI based stability analysis and controller design for a class of 2D discrete linear systems, Proceedings of 40th IEEE Conference on Decision and Control, 2001, pp. 4457-4462. 